\title{
D’une hagionymie abondante à une hiéronymie supplétive.
} Usage d'une stratégie toponymique chez les Franco-Canadiens de la Saskatchewan

\section{From Abundant Hagionymy to Dedicatory Suppletive Hieronymy. A Toponymic Strategy Used by Franco-Canadians in Saskatchewan \\ De una abundante hagionimia a una hieronimia supletoria. Uso de una estrategia toponímica por los Franco-Canadienses de la Saskatchewan}

\author{
Carol J. Léonard
}

Volume 54, numéro 151, avril 2010

URI : https://id.erudit.org/iderudit/044365ar

DOI : https://doi.org/10.7202/044365ar

Aller au sommaire du numéro

Éditeur(s)

Département de géographie de l'Université Laval

ISSN

0007-9766 (imprimé)

1708-8968 (numérique)

Découvrir la revue

Citer cet article

Léonard, C. J. (2010). D’une hagionymie abondante à une hiéronymie supplétive. Usage d'une stratégie toponymique chez les Franco-Canadiens de la Saskatchewan. Cahiers de géographie du Québec, 54(151), 25-40. https://doi.org/10.7202/044365ar

\section{Résumé de l'article}

La toponymie participe à l'affirmation de l'identité chez nombre de groupes dont les territoires se jouxtent ou partagent un même espace. Chez les groupes en contact avec d'autres langues et cultures que la leur, l'affirmation des spécificités linguistiques et culturelles en toponymie peut parfois se révéler délicate. Pour nommer les lieux, les humains disposent d'un arsenal de stratégies dénominatives, tant pour faire étalage des spécificités de leur groupe d'appartenance que pour dissimuler des particularismes susceptibles d'attirer sur eux une attention non recherchée. Dans cet article, les exemples sont tirés d'un répertoire de près de 2500 toponymes d'origine et d'influence françaises en Saskatchewan. Alors que l'hagionymie catholique et française abonde dans cette province au cours de la période qui précède celle de la colonisation, elle se raréfie remarquablement, toutes proportions gardées, dès les premières décennies du $\mathrm{XX}^{\mathrm{e}}$ siècle. Pour pallier les difficultés que représente ce mode de dénomination identitaire, on a eu recours, en certains endroits, à une hiéronymie dédicatoire supplétive. 


\title{
D'une hagionymie abondante à une hiéronymie supplétive.
}

\section{Usage d'une stratégie toponymique chez les Franco-Canadiens de la Saskatchewan}

\author{
From Abundant Hagionymy to Dedicatory \\ Suppletive Hieronymy. A Toponymic \\ Strategy Used by Franco-Canadians in \\ Saskatchewan
}

De una abundante hagionimia a una

hieronimia supletoria. Uso de una

estrategia toponímica por los Franco-

Canadienses de la Saskatchewan

\author{
Carol J. LÉONARD \\ Université de l'Alberta
}

Carol.Leonard@ualberta.ca

\section{Résumé}

La toponymie participe à l'affirmation de l'identité chez nombre de groupes dont les territoires se jouxtent ou partagent un même espace. Chez les groupes en contact avec d'autres langues et cultures que la leur, l'affirmation des spécificités linguistiques et culturelles en toponymie peut parfois se révéler délicate. Pour nommer les lieux, les humains disposent d'un arsenal de stratégies dénominatives, tant pour faire étalage des spécificités de leur groupe d'appartenance que pour dissimuler des particularismes susceptibles d'attirer sur eux une attention non recherchée. Dans cet article, les exemples sont tirés d'un répertoire de près de 2500 toponymes d'origine et d'influence françaises en Saskatchewan. Alors que l'hagionymie catholique et française abonde dans cette province au cours de la période qui précède celle de la colonisation, elle se raréfie remarquablement, toutes proportions gardées, dès les premières décennies du $\mathrm{XX}^{\mathrm{e}}$ siècle. Pour pallier les difficultés que représente ce mode de dénomination identitaire, on a eu recours, en certains endroits, à une hiéronymie dédicatoire supplétive.

\section{Mots-clés}

Toponymie, hagionymie, hiéronymie, identité, stratégie, minorité.

\begin{abstract}
Toponymy plays an important role in declaring identity among groups with adjoining territories as well as with those sharing the same space. Affirmation of cultural and linguistic identity through toponymy can sometimes prove to be a delicate undertaking, however. Groups in contact with languages and cultures other than their own have at their disposal an array of denominative strategies both to highlight the unique features of the group to which they belong, and to conceal aspects likely to attract unwanted attention. The examples quoted in this article are drawn from a repertoire of close to 2500 toponyms, of native and French origin, from Saskatchewan. Although Catholic and French hieronymy was prominent in Saskatchewan during
\end{abstract}


the period before colonization, it had - relatively speaking - declined significantly by the turn of the twentieth century. To offset the difficulties inherent to this kind of identity-bound denomination, in certain instances a dedicatory suppletive hieronymic approach was prefered.

\section{Keywords}

Toponymy, hagionymy, hieronymy, identity, strategy, minority.

\section{Resumen}

La toponimia toma parte en la afirmación de la identidad de numerosos grupos cuyos territorios se yuxtaponen o que comparten el mismo espacio. Para los grupos que están en contacto con otras lenguas y culturas que la suya, la afirmación de especifidades lingüísticas y culturales en toponimia puede ser difícil. Para denominar lugares, los humanos disponen de todo un arsenal de estrategias denominativas, sea para hacer ostentación de aspectos específicos del grupo de pertenencia o para disimular particularismos susceptibles de llamar una atención no deseada. En este artículo se presentan ejemplos de un repertorio de 2500 topónimos de origen y de influencia francesa en Saskachewan. Mientras que la hagionimia católica y francesa abunda en esta provincia durante el periodo que precede al de la colonización, se observa su rarefacción, teniendo en cuenta las proporciones, durante los primeros decenios del siglo XX. Para superar las dificultades de ese modo de denominación identitaria, se recurre, en ciertos lugares, a una hieronimia dedicatoria y supletoria.

\section{Palabras clave}

Toponimia, hagionimia, hieronimia, identidad, estrategia, minoría.

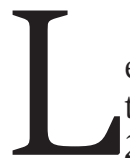

es noms qu'un peuple donne aux lieux qu'il fréquente participent à l'affirmation de son identité groupale et territoriale (Giraut et Houssay-Holzschuch, 2008; Nash, 1999; Woerhling, 1998). Ils forment ce qu'on pourrait appeler un référentiel toponymique identitaire. Il ne faut donc pas s'étonner du grand soin parfois mis à l'édification de cette toponymie. «Symboles de l'habiter, leurs noms de lieux concrétisent et singularisent les identités culturelles» (Adam, 2008: 337). Étudier la toponymie d'un groupe culturel (ses noms de lieux), c'est emprunter une voie permettant de saisir les multiples manières avec lesquelles, en s'inscrivant dans le territoire, un groupe a voulu s'orienter, mais aussi se définir et, parfois même, être perçu. Car le toponyme joue un double rôle. Il est, par sa nature, une enseigne définitoire qui permet de localiser une entité géographique. Il est aussi une bannière sur laquelle on inscrit une manière d'être soi-même, de voir la nature, d'afficher ses croyances, de chérir les siens ou son passé. Cette seconde fonction confère au nom de lieu son attribut culturel, ce qui autorise à regarder la toponymie comme un véritable mode d'expression identitaire (Dorion et Beaupré, 1994).

Dans les régions multilingues, la toponymie ne fait pas que refléter l'état du partage d'un territoire entre les groupes linguistiques et culturels. Elle peut aussi marquer l'état des rapports entre les groupes partageant ou se disputant la région qu'ils habitent (Jenson, 1993 ; Berg et Kearns, 1996; Light et al., 2002 ; De la Soudière, 2004). Dans un contexte de contact des langues et des cultures, l'affirmation des spécificités linguis- 
tiques et culturelles peut parfois se révéler délicate et entravée. Certaines pratiques telles que l'emploi exclusif de génériques dans une langue unique (Albert Lake plutôt que Lac Albert, Court Lake plutôt que Lac Court), l'omission de signes diacritiques parfois essentiels à une interprétation juste (Page Lake ou Dube Lake plutôt que Pagé Lake ou Dubé Lake) en constituent des exemples (Léonard, 2010). Ainsi, certains aménagements toponymiques peuvent entraîner une perte des repères essentiels à l'association d'un nom de lieu à la langue et à la culture qui l'a produit.

Au cours de son histoire, la francophonie saskatchewanaise a fait usage de divers procédés dénominatifs afin de s'entourer d'une toponymie à son image. Ainsi, elle a nommé les lieux en référence à des pionniers (Courval, Coderre, Lepine, Ville Bouvier, Zenon Park). Elle a emprunté à la France et au Québec des noms qu'elle a transplantés sur le sol saskatchewanais (Bourgonne, Domrémy, Ponteix, Quimper). Elle a aussi eu recours à des vocables que les spécialistes des noms géographiques nomment les hagiotoponymes ou, plus commodément, les hagionymes, c'est-à-dire les noms de lieux formés, en tout ou en partie, de noms de saints ou de saintes (Bonne Madone, Saint-Brieux, Saint-Jacques de Dauphinais, etc.).

Tout au long des paragraphes qui suivent, nous dépeindrons brièvement le contexte évolutif de l'hagionymie saskatchewanaise depuis l'arrivée des missionnaires catholiques. Nous présenterons celle qui a fleuri sous l'action de leur apostolat. Nous la mettrons en contraste avec celle qui est apparue au cours de la colonisation et avec celle du Québec, principal foyer du peuplement francophone de l'Ouest canadien. Nous évoquerons les tensions linguistiques et religieuses pour expliquer, en partie tout au moins, la rareté relative de l'hagionymie dans le centre-sud de la Saskatchewan. Nous présenterons enfin deux procédés, l'hagionymie tronquée et l'hiéronymie ${ }^{1}$ comme méthodes pour maintenir la représentation de la foi catholique dans la toponymie de cette région.

\section{Une présence française affirmée}

Au moment de l'arrivée des Européens, le territoire provincial est occupé par des habitants appartenant à trois groupes linguistiques distincts qui s'expriment en langues athapascanes (Chipewyans, Castors et Esclaves), algonquiennes (Cris et Pied-Noirs) et siouses (Assiniboines et Gros-Ventres). Les premiers Blancs à s'aventurer sur le territoire qui forme aujourd'hui l'Ouest canadien parlent en majorité le français. Les premiers comptoirs de traite bâtis par eux sur la rivière Saskatchewan apparaissent peu avant la cession de la Nouvelle-France à la Couronne britannique. Par la suite, la population francophone de la vallée laurentienne continue de participer activement au commerce des fourrures et fournit un nombre considérable d'engagés de la traite que l'on nomme aussi les «voyageurs». En 1786, on estime leur nombre à plus de 400 sur l'ensemble du territoire. Les Métis d'expression française, fils et filles de l'union des engagés de la traite avec des Amérindiennes, donnent naissance aux principaux noyaux de peuplement francophone en Saskatchewan: régions de Batoche, de Qu'Appelle et de la montagne de Bois (Wood Mountain).

1 Le terme «hiéronymie» désigne ici tout nom de lieu destiné à reconnaître le caractère sacré ou le mérite d'une vocation entièrement consacrée au service de Dieu (ex. : les noms d'entités honorant des Oblats : Mazenod, Little Faraud Lake, Little Grollier Lake, Little Taché Lake et Marchand Lake). 
Le 15 juillet 1870 marque le transfert effectif des terres incluses dans la charte de la Compagnie de la Baie d'Hudson à la Couronne d'Angleterre. La colonisation de l'Ouest du Canada est alors mise en branle. Afin de résoudre des problèmes de nature postale, l'administration fédérale canadienne crée, en 1882, quatre districts provisoires qu'elle taille à même ces territoires : l'Alberta, l'Athabasca, l'Assiniboia et la Saskatchewan. Ces deux derniers districts, une fois réunis en 1905, forment une province qui prend le nom de Saskatchewan. Cette province est constituée d'un territoire dont on peut dire qu'il est «tout intérieur» et qu'il possède des frontières dont les tracés sont entièrement artificiels. Elle est bornée au $49^{\mathrm{e}}$ parallèle par les États-Unis et au $60^{\mathrm{e}}$ parallèle par les Territoires du Nord-Ouest, à l'est par le Manitoba et à l'ouest par l'Alberta.

La colonisation des terres qui forment aujourd'hui l'écoumène agricole de la Saskatchewan s'engage lentement au cours des années 1880. Au début de cette période, l'élément français représente environ la moitié de la population d'extraction européenne (Lalonde, 1983: 82). Puis, la colonisation connaît une accélération au tournant du $\mathrm{XX}^{\mathrm{e}}$ siècle. Les francophones de l'est du pays, si présents aux beaux jours du commerce des fourrures, ne répondent pas avec empressement à l'appel du peuplement de l'Ouest. En 1911, alors que la Saskatchewan compte 490432 habitants, seulement 25497 affirment des origines françaises (5,2\%).

\section{Une hagionymie missionnaire abondante}

Le fer de lance de l'évangélisation dans l'Ouest, comme le rappelle Choquette (1995 : 29), est d’abord le clergé séculier. On s’appuie sur lui au cours de la période qui s'étend de 1818 à 1845 . Le territoire est si vaste que les quelques prêtres doivent se contenter d'exercer leur ministère auprès de la population de la mission de la rivière Rouge. En 1845, les premiers missionnaires Oblats de Marie-Immaculée font leur apparition. Sitôt débarqués, ils se consacrent principalement à la fondation de missions autochtones. Celles de la Saskatchewan apparaissent d'abord le long des anciennes routes empruntées par les traiteurs de fourrures. La toute première mission permanente, nommée Saint-Jean-Baptiste, est fondée à l'Île-à-la-Crosse en 1846 grâce à deux missionnaires: Alexandre-Antonin Taché et Louis-François Richer-Laflèche. Puis, en 1853, apparaît la mission Notre-Dame-des-Sept-Douleurs aux confins du lac Athabasca, à Fond-du-Lac. En 1858, on crée celle de Notre-Dame-de-la-Visitation au lac La Loche et, en 1860, celle de Saint-Julien au lac Vert.

La fondation de ces missions marque le début de l'action prosélytique de l'Église catholique dans les régions septentrionales saskatchewannaises. Elle marque également celui de l'usage intensif d'hagionymes, signes de l'expansion du fait français et catholique en ces lointaines contrées. Les hagionymes des missions et dessertes reportés à la figure 1 et l'analyse de leurs motifs de dénomination ne laissent pas suspecter la réponse des missionnaires à un modèle de dénomination préétabli. L’hagionyme choisi est parfois celui du saint du jour au calendrier. Une autre mission prend le nom du saint patron du missionnaire fondateur ou celui d'un membre de la famille ou encore celui d'un généreux donateur. Les sources d'inspiration sont nombreuses ; le martyrologe est vaste. Toutefois, l'une d'elles semble exercer un attrait particulier puisque près du quart des hagionymes missionnaires (23\%) réfèrent de manière explicite à la Vierge Marie. Voici une liste qui permet d'en juger : 


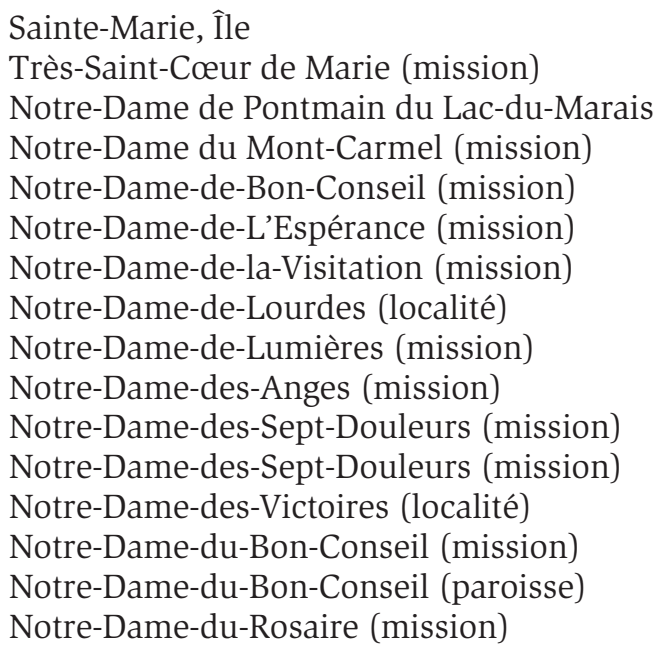

Les missionnaires de l'Ouest canadien sont, il est vrai, en vaste majorité des Oblats de Marie-Immaculée.

En 1868, à la vieille de la création du Manitoba, on assiste à une refonte de la carte archidiocésaine et le vicariat de la Saskatchewan est créé. La véritable colonisation de l'Ouest va bientôt débuter.

\section{La colonisation, une déferlante toponymique}

En 1867, le Canada - nouveau-né - vit déjà un malaise économique profond. Pour sortir du marasme, le gouvernement décide de mettre en œuvre une politique nationale dont le peuplement de l'Ouest est l'un des fers de lance. La construction d'un chemin de fer transcontinental en est un autre. Tous deux sont indissociables. Les immenses prairies ainsi peuplées, grouillantes d'une population active et besogneuse, vont rassembler le lot de consommateurs dont les industries canadiennes ont tant besoin pour prospérer à nouveau. Comme nous l'avons souligné, au début de cette période, l'élément français représente environ la moitié de la population d'extraction européenne présente sur le territoire qui va devenir la Saskatchewan. Avec l'afflux ininterrompu d'immigrants anglo-saxons, l'équilibre se rompt. L'épiscopat de l'Ouest décide au plut tôt de prendre en main la colonisation catholique et française. Il la dominera (Lalonde, 1983 : 82). En fait, pendant toute la période que dure la colonisation de la Saskatchewan, «de 1870 à 1930, la très forte majorité des membres de l'élite française des Prairies [porte] une soutane» (Ibid.). Malgré tous les efforts du clergé, malgré les appels au renfort faits auprès des Canadiens français du Québec et de ceux qui s'étaient exilés en Nouvelle-Angleterre, malgré les efforts de recrutement en France et en Belgique, la population catholique et française de l'Ouest est rapidement mise en minorité (Painchaud, 1986: 72). On ne parvient à assurer une concentration de groupes francophones qu'en certains endroits. 
Figure 1 Missions oblates en Saskatchewan pour les périodes du $\mathrm{XIX}^{\mathrm{e}}$ et $\mathrm{XX}^{\mathrm{e}}$ siècles

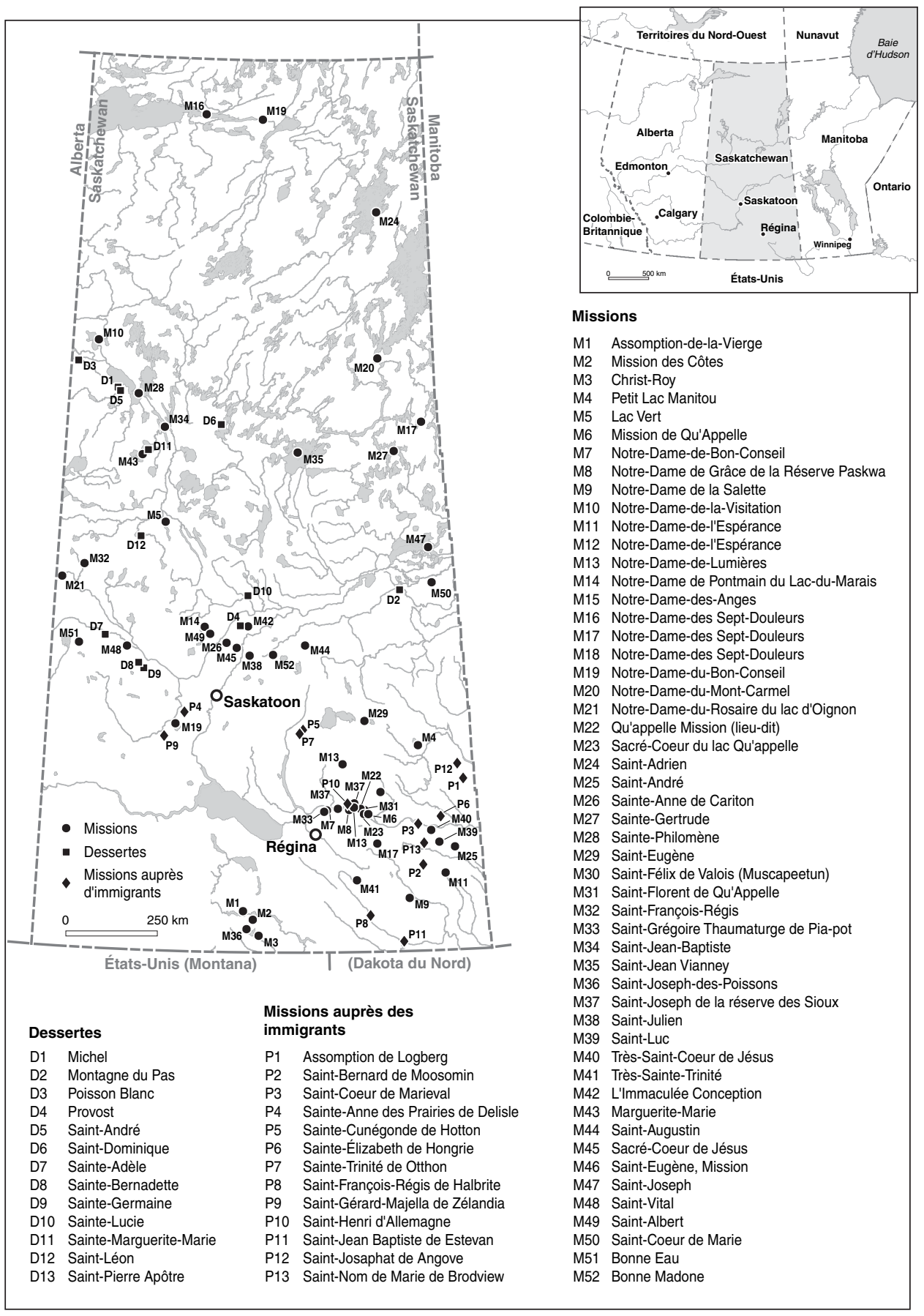


La période de la colonisation est celle de tous les bouleversements. Le territoire change de statut, est découpé, fractionné par son cadastre, envahi et dominé. Il se constelle d'une multitude de noms nouveaux. La compagnie de chemins de fer Canadien Pacifique, qui se voit confier la construction de la voie ferrée transcontinentale, a alors une influence marquante sur la manière de nommer les lieux, souligne le toponymiste Bill Barry. Or, les noms auxquels elle a le plus recours sont les anthroponymes. Viennent ensuite les transplantations de noms empruntés à d'autres régions du pays et du monde (Barry 1997 : 49). L'hagionymie est pour ainsi dire absente le long des voies ferroviaires. Elle est peu présente ailleurs également. En fait, on constate l'absence presque totale d'une hagionymie populaire saskatchewanaise inscrite dans le cadre civil.

Des 12500 noms contenus dans le Répertoire toponymique du Canada consacré à la Saskatchewan (1998), seule une quarantaine, soit 0,003\%, contiennent les épithètes «saint» ou "sainte», sous leur forme complète ou abrégée. Ce chiffre n’est atteint que parce qu'il y a aussi une déclinaison locale de plusieurs hagionymes: St. Louis côtoie St. Louis №431 (municipalité rurale). St. Victor (village) jouxte St. Victor Petroglyphs Provincial Park. Val Marie s’aligne aux côtés des Val Marie №17 (municipalité rurale) et Val Marie Reservoir Migratory Bird Sanctuary. Plus de la moitié de ces noms n'entretiennent pas de véritables rapports avec la langue française. Seuls six d'entre eux portent le tiret, caractéristique graphique de l'hagionymie française. Voici la liste complète:

St. Aldwyn, St. Alphege, St. Andrews №287, St. Antoine, St. Benedict, St. Boswells, St. Brieux, St. Brieux Regional Park, Saint Cyr Hills Trails Recreation Site, St. Cyr Lake, Saint Cyr Lake, St-Denis, St. Elmo Lake, Ste-Marthe-Rocanville, Saint-Front, St. George's Hill, St. Germain Lake, St. Gregor, Saint Hippolyte, St. Hubert Mission, St. Isidore-de-Bellevue, St. John Lake, St. Joseph's, Saint Lake, St-Laurent-Grandin, St. Louis, St. Louis №431, St. Luke, St. Marys Channel, St. Peter Game Preserve, St. Peter №369, St. Peters Colony, St. Philips, St. Philips №301, St. Pierre Lake, St. Victor, Saint Victor Petroglyphs Provincial Park, St. Walburg, St. Walburg Recreation Site, Vallée-Ste-Claire.

En outre, certains des toponymes parés de l'épithète «saint» ou «sainte» se révèlent être de faux hagionymes puisqu'ils sont en fait des patronymes familiaux. C'est le cas des toponymes St. Pierre Lake et St. Cyr Lake².

En Saskatchewan, bien entendu, la majorité de la population est anglo-protestante, et les anglo-protestants pratiquent peu l'hagionymie. Entre 1911 et 1921, la proportion des habitants de foi catholique romaine varie de 16 à $20 \%$ de la population. L'hagionymie n'y est donc pas courante. Le père Adrien-Gabriel Morice, auteur d'une histoire de l'Église catholique dans l'Ouest, attribue cette rareté hagionymique à «l'administration civile et aux compagnies ferroviaires» (1921: III, 454) plus enclines à nommer d'autres manières.

2 En mémoire du soldat Armand L.V. St-Pierre (Saskatchewan Archives Board, R-73.II. Mayson, Richmond, volume 3) et en l'honneur d'Arthur Saint-Cyr, arpenteur-géomètre (Saskatchewan Archives Board, R-183 I.458 Saint-Cyr). 
Si, dans son ensemble, l'hagionymie saskatchewanaise se révèle relativement rare, il y a tout de même lieu d'examiner les noms de lieux où réside la population francocatholique saskatchewanaise.

Dans leur monographie intitulée Histoire des Franco-Canadiens de la Saskatchewan (1986), Lapointe et Tessier identifient 99 lieux habités où les francophones forment «la majorité ou la minorité la plus forte».

Admiral, Alida, Albertville, Arborfield, Assiniboia, Batoche, Bellegarde, Bellevue, Beynes, Big River, Bonne Madone, Butte Saint-Pierre, Cabana, Cadillac, Cantal, Carlton, Celtic, Chambéry, Clémenceau, Coderre, Courval, Debden, Delmas, Dollard, Domrémy, Duck Lake, Dumas, Edam, Ferland, Forget, Frenchville, Gouverneur, Gravelbourg, Henribourg, Hoey, Jacklish Lake, High View, Lac Lenore, Lacordaire, Lac Pelletier, Lac Vert, Laflèche, Lajord, La Plaine, Laventure, Leask, Lépine, Leovllle, Lisieux, Makwa, Marcelin, Meota, Yronne, Milly, Montmartre, Moose Jaw, Nipawin, Nobleville, North Battleford, Ormeaux, Oxbow, Paradise Hill, Pascal, Périgord, Ponteix, Pré Sainte-Marie, Prince, Prince-Albert, Prud'homme, Quimper, Radville, Redvers, Regina, Reynaud, Rosetown, Rose Valley, Royer, Saint-Brieux, Saint-Denis, Saint-Front, Saint-Hubert, Saint-Hippolyte, Saint-Louis, Saint-Victor, Sainte-Marthe de, Saskatoon, Sedley, Souris Valley, Spiritwood, Storthoaks, Val-Marie, Vawn, Veillardville, Victoire, Vonda, Wakaw, Wauchope, Willow-Bunch, Zenon-Parc (Ibid. : 117).

Au nombre de ces 99 localités, 62 possèdent des toponymes d'origine ou d'influence françaises. Près de $73 \%$ de ceux-ci, hagionymes inclus, sont constitués d'anthroponymes. Sur l'ensemble des 99 toponymes, on ne dénombre que 12 hagionymes: Bonne Madone, Butte Saint-Pierre, Pré Sainte-Marie, Saint-Brieux, Saint-Denis, Saint-Front, Saint-Hubert, Saint -Hyppolyte, Saint-Louis, Saint-Victor et Sainte-Marthe de Rocanville. Il est à noter que l'hagionyme Saint-Isidore-de-Bellevue y est représenté sous sa forme abrégée Bellevue. L’hagionymie seule représente donc environ $20 \%$ des noms de la liste qui sont (pour aller au plus court) des noms de lieux français.

Cette liste ne rend pas entièrement compte de l'hagionymie créée par les FrancoSaskatchewanais. Il existe aussi une hagionymie qu'on peut qualifier de «scolaire» et qui appartient, il faut le préciser, davantage au passé qu’au présent. Les premiers arrondissements scolaires apparaissent en Saskatchewan vers 1880. Ces petits territoires constituent des délimitations de fréquentation scolaire. Leur superficie ne doit pas dépasser les 20 milles carrés. Au cours des cinq décennies qui sont celles de la colonisation, il s'en crée plus de 5000. De ce nombre, on peut en identifier une quarantaine dont les noms sont à caractère hagionymique $(0,008 \%)$ et pour lesquels la graphie, le lieu d'apparition et les motifs de désignation permettent de les associer aux colons francophones. En voici quelques exemples: Sacre-Coeur S.D. №1586, Saint Alexis S.D. №4522, St. Eloi S.D. №3840, St. François de Taché R.C.S.D. №16, St. Vital R.C.P.S.D. №11.

Ces petits arrondissements sont souvent isolés dans la prairie et leur petite école ne comporte, dans nombre de cas, qu'une seule salle de classe dans laquelle s'entassent les élèves tous âges confondus. Ces arrondissements répondent difficilement aux besoins en éducation de l'époque. Au début des années 1940, l'augmentation des coûts liés à l'instruction, les disparités régionales, mais aussi l'amélioration des moyens de transport et les progrès technologiques, conduisent le gouvernement provincial 
à prendre des mesures pour moderniser le système scolaire. En 1944, le gouvernement provincial adopte le Larger School Unit Act qui va lui permettre de regrouper les arrondissements scolaires en unités plus étendues. Cette mesure fait perdre aux francophones de certaines régions le contrôle qu'ils avaient jusque-là sur leurs écoles paroissiales. Ceux-ci deviennent minoritaires au sein des grandes unités scolaires et les commissaires francophones élus ne peuvent plus assurer la langue de communication utilisée par les enseignants dans leurs écoles sans l'aval des commissaires scolaires régionaux, pour la plupart anglophones. Avec la centralisation progressive, disparaît de la sphère publique officielle cette hagionymie scolaire. Enfin presque, puisqu'on la retrouve dans les très nombreuses monographies consacrées aux régions, aux villes et aux villages. Il est important de souligner combien est révélatrice la manière dont un grand nombre de ces monographies sont rédigées. En effet, c'est très souvent dans les sections du livre réservées aux anciens arrondissements scolaires qu'on retrouve les histoires de chacune des familles locales. Cette disposition affirme en quelque sorte le rôle majeur à la fois de repère et de milieu de vie que représentaient les petits arrondissements et qu'ils représentent encore aujourd'hui pour ceux qui les ont connus.

\section{Montée de l'intolérance}

Dresser un bilan de l'hagiotoponymie saskatchewanaise, tant à l'échelle provinciale $(0,0003 \%)$ qu'à l'intérieur des principaux foyers de peuplement francophone (11\%), demeure une tâche délicate. On peut soutenir que, parmi les plus habilités à le faire, il y a ceux-là même qui avaient charge et pouvoir de nommer les lieux. Les noms qui tapissent les cartes contemporaines de la province ayant pour la plupart été donnés au plus fort de la colonisation, c'est-à-dire il y a maintenant près d'un siècle, les principaux témoins sont aujourd'hui disparus. Trouvèrent-ils satisfaction? Sont-ils parvenus à nommer comme il le désiraient? La présence à l'avant-scène de l'Église catholique tout au long de la colonisation mène naturellement à penser que l'hagionymie des localités à prédominance francophone pouvait être encore plus abondante, plus affirmée. En situation de contact des langues et des cultures, tout bilan exige une exploration des relations, une prise en compte du climat ambiant.

À l'aube du XXe siècle, la Saskatchewan connaît une croissance démographique prodigieuse. Sa population quintuple entre 1901 et 1911. On favorise l'immigration britannique certes, mais également celle en provenance d'Allemagne, de Scandinavie, des Pays-Bas, de Belgique et enfin de France. Dès 1901, la proportion de la population d'origine européenne autre que britannique représente $36 \%$. L’arrivée massive d'éléments disparates, qui de surcroît ne peuvent pour une bonne part s'exprimer en langue anglaise, est de nature à en inquiéter certains (Lapointe et Tessier, 1986). Leur assimilation, croit-on alors, peut et doit se faire. Une certaine élite souhaite s'assurer que la nouvelle province ne puisse être autre qu'anglo-saxonne. L'éducation, fer de lance du maintien de l'homogénéité culturelle, est perçue comme le meilleur moyen de préserver la cohésion sociale (Huel, 1978).

En 1906, un inspecteur d'école manitobain présente, sans détour ni nuance, ce qui à ses yeux doit être le rôle du système scolaire public: 
The great work of the public school in Canada is the formation and development of a high type of national life. This is particularly true in Western Canada, with its heterogeneous population. Here are to be found people of all countries, from the keen, clever American, with highly developed national ideals, equal to but perhaps somewhat antagonistic to our own, to the ignorant peasantry of central and Eastern Europe and Asia. These incongruous elements have to be assimilated, have to be welded into one harmonious whole if Canada is to attain the position that we, who belong here by right of birth and blood, claim for her. The chief instrument in this process of assimilation is the public school. (Maguire, 1906:31)

L'éditorial du Winnipeg Free Press du 8 juillet 1898 va dans le même sens en avançant que: "Canada is Anglo-Saxon and will remain Anglo-Saxon. Foreigners may come in their thousands, and they, too, if not in the first, then in the second generation, will also be Anglo-Saxon» (Barber, 1972: xvi-xvii). Ce faisant, on inclut les Canadiens français au nombre des étrangers. Plus d'un sont d'opinion que les «French», loin d'être des partenaires dans la Confédération, sont simplement «another ethnic group speaking another foreign language» (Thompson, 1978: 74).

Dans plusieurs milieux, on considérait les Canadiens français comme des étrangers; puisqu'ils tenaient à préserver leur identité ethnique par l'enseignement de la langue ancestrale, les écoles publiques sous leur contrôle allaient devenir la cible rêvée de tous ceux qui aspiraient à remettre le système d'enseignement public sur le «droit chemin».

(Huel, 1983a: 203)

Les années 1910 sont marquées par une controverse touchant les écoles confessionnelles. Bien qu'une animosité couve depuis la création de la province en 1905 (Huel, 1983b), c'est en 1912 que les Conservateurs provinciaux font connaître leur intention de tout faire pour interdire l'enseignement d'une autre langue que l'anglais. Les francophones sont directement visés. Pendant la Première Guerre mondiale, les tensions s'exacerbent. La position du Québec face à la conscription soulève, à l'époque, les passions de certains zélateurs protestants qui, comme le pasteur Murdock MacKinnon, veulent constamment en découdre avec les Canadiens français. «French must go [since] Quebec failed us during the war. We do not want Quebec reproduced in Saskatchewan...» lance ce dernier. «Let all enlightened citizens speak, write, and wire until French goes with German...» (Huel, 1978:301).

Le climat est tel que le haut clergé se sent lui-même contraint d'agir avec discrétion. La crainte et le souci se devinent chez Mgr Olivier-Elzéar Mathieu (évêque de Regina en 1911, puis archevêque en 1917) lorsqu'il fait allusion à l'institution qu'il rêve de fonder à Gravelbourg: "Si je pouvais sans bruit, disait-il, fonder ce collège avant de mourir, je croirais avoir fait l'œuvre de ma vie parce que j’aurais sauvé la langue française dans toute la province pour toujours...» (Lalonde, 1983 : 90) ${ }^{3}$. Ce même tact, il l'obtient des associations provinciales et du journal Le Patriote de l'Ouest alors que l'Ontario français est soumis au Règlement 17 afin «de ne pas jeter de l'huile sur le feu» (Huel, 1983b : 319).

Le Parti conservateur provincial n'a de cesse de poursuivre son ambition d'abolir l'enseignement en français jusqu'à ce qu'il accède au pouvoir en 1929 avec le soutien des loges orangistes et du Ku Klux Klan dont les actions d'intimidation à l'endroit

3 Le prélat réalisa son vœu en 1917 lorsqu'il cofonda le collège catholique de Gravelbourg qui, aujourd’hui encore, porte son nom. 
des catholiques francophones se remarquent particulièrement dans le sud de la province (Lapointe et Tessier, 1986). Le 27 février 1931, le premier ministre conservateur J.M.T. Anderson présente un amendement à la loi scolaire. Le français cesse d'être une langue d'enseignement.

Il va sans dire qu'un tel climat n'est alors pas favorable à l'expression/expansion du modèle français québécois et que le marquage toponymique du territoire par les francophones risque d'en avoir porté les stigmates.

Le ministère des Postes, par exemple, aura par moment exprimé clairement son refus d'adopter des hagionymes, de crainte que des toponymes quasi homographes (dont la graphie est semblable) soient à la source de confusion et entravent la bonne conduite du service de la poste. En témoignent certaines pétitions et protestations dont on retrouve des traces dans des monographies régionales. La petite histoire de la naissance du nom Ferland, donné à un hameau situé au sud de la province, en fournit un exemple.

Originaires de la paroisse de Sainte-Claire, dans le comté de Dorchester au Québec, les premiers colons de l'endroit souhaitent perpétuer le souvenir de leur lieu d'origine. Le 3 juillet 1910, les intéressés, avec à leur tête Edmond Chabot, s'adressent à Rodolphe Lemieux, ministre des Postes d'alors, afin d'obtenir un bureau de poste baptisé Ste-Claire-des-Prairies. Du fait de l'existence d'un bureau au Québec désigné sous le nom de Vallée Sainte-Claire, les autorités optent pour une désignation abrégée: Des Prairies.

\footnotetext{
Comme il fallait s'y attendre, le nom «Des Prairies» ne signifiant rien, ne plut pas aux pionniers. On en demanda un autre. Le 22 mai 1911, Edmond Chabot, maître de poste, demandait que le nom «Des Prairies» soit changé en celui de St-Edmond, vu qu'il existait déjà en Saskatchewan un bureau connu sous le nom de «Lac des Prairies».

Le nom «Saint-Edmond» ne fut pas accepté parce qu'il y avait déjà une localité connue sous ce nom. L'honorable Rodolphe Lemieux, de lui-même, décida d'appeler ce bureau «Ferland» et data ce changement le premier juillet 1911. Le ministre des Postes, en baptisant le bureau de poste du nom de Ferland, écrivait aux intéressés et leur dit que c'était le nom d'un grand historien canadien-français, l'abbé Jean-Baptiste Ferland. (Chabot, 1961: 17-18)
}

Le 25 novembre 1911, mécontents de se voir imposer un nom, les résidants remontent à l'assaut avec une nouvelle requête dans laquelle ils expriment le vœu de voir la localité être nommée Chabotville. Le gouvernement répond qu'une politique interne lui interdit d'employer les noms de personnes vivantes. La population revient à la charge et propose cette fois Sainte-Marie des Prairies. Cette demande demeure sans réponse.

En d'autres circonstances, les tensions sont assez apparentes pour expliquer l'exclusion de certains noms au profit d'autres. L'hagionyme Ste-Hélène ne peut ainsi être retenu en raison d'un désaccord entre les résidants. Le médecin de la localité propose le nom qui deviendra officiel, Prud'homme, en l'honneur Joseph-Henri Prud'homme, premier prêtre natif de l’Ouest à se voir élevé à la dignité épiscopale.

Les motifs de l'éviction partielle ou totale des hagionymes ne sont pas toujours exprimés clairement. Les données manquent pour faire toute la lumière sur les motifs à l'origine de changements suivants: Sainte-Thérèse-de-Lisieux qui devint Lisieux 
ou Notre-Dame-des-Victoires qui devint Victoire. À quoi doit-on le remplacement de Saint-Antoine-des-Prairies par Storthoaks, celui de Notre-Dame d'Auvergne par Ponteix et celui de Saint-Florent-de-Qu'Appelle par Lebret?

\section{Recours à l'hiéronymie supplétive}

l'hagiotoponymie française n'est pas l'unique moyen d'afficher sur la carte une appartenance à la culture française et un attachement à la foi catholique et romaine. Le recours à l'hiéronymie, en l'occurrence l'emploi, par exemple, de patronymes empruntés à des figures importantes de l'Église, permet d'atteindre ce résultat. À la différence de l'hagionyme, l'hiéronyme est habituellement dépourvu des épithètes «saint» et «sainte» qui constituent des références explicites au domaine religieux. Il offre donc un aspect plus discret. Son association à la sphère religieuse est moins immédiate.

On trouve de tels hiéronymes concentrés en un endroit de la province, le centre-sud, plus particulièrement dans la région immédiate de Gravelbourg, ville que d'aucuns ont élevée au rang de centre provincial de la francophonie saskatchewanaise. Ces hiéronymes sont au nombre de quatre et leur création, au cours des deux premières décennies du siècle dernier, est attribuée à l'abbé Louis-Pierre Gravel, prêtre-colonisateur influent et fondateur de Gravelbourg. Il s'agit de Gravelbourg, Lafleche, Mazenod et Plessis (figure 2).

De l'aveu même du fondateur de cette ville, Gravelbourg fut nommée en hommage à Elphège Gravel (1838-1904), évêque de Nicolet et cousin de Louis-Pierre Gravel ${ }^{4}$. Lafleche rappelle le souvenir de Louis-François Richer dit Laflèche (1818-1898), missionnaire de l'Ouest (Royer, 1908) qui devint évêque de Trois-Rivières. La localité éphémère de Plessis fut ainsi nommée en souvenir de Joseph-Octave Plessis (1763-1825), premier archevêque de Québec. Enfin, Mazenod doit son nom à la mémoire de Charles-Joseph-Eugène de Mazenod (1782-1861), évêque de Marseille et fondateur des Oblats (Carrière, 1958).

À propos du nom Mazenod, le père Morice relève ce commentaire de l'abbé Gravel: "C'est le nom d'un grand chef, disait-il finement aux Anglais curieux d'apprendre ce que représentait cet étrange vocable» (1928: IV, 79, n.18). Dans une région dominée quelques décennies auparavant par les Amérindiens, on comprendra ici que le père Morice laisse entendre que l'abbé Gravel a intentionnellement voulu renvoyer ses interlocuteurs protestants à une réalité autre que celle qu'il affirmait caractériser.

Pour mieux mettre en perspective cette hiéronymie, notons d'une part, qu'on ne retrouve que deux hagionymes officiels et aisément identifiables dans le sud de la province: St.Hubert Mission et St.Victor. D’autre part, deux autres noms de lieux, situés également au sud de la province, sont des hagionymes qui ne se révèlent pas au premier abord. Il s'agit de Marieval et de Val Marie. Dans l'un et l'autre cas, le prénom Marie est emprunté à la Mère du Christ (Morice, 1921-1923 ; Barry, 1998).

4 Propos recueillis en octobre 1990 par l'auteur auprès de madame Lucienne Gravel de Montréal, auteure et biographe de la famille Gravel. 
Le climat difficile pour les Franco-catholiques au cours des premières décennies du siècle dernier, l'action discrète qui semble favorisée par le clergé et les marques de dissimulation dans la toponymie hagionymique mariale semblent indiquer que la présence du groupe d'hiéronymes dans le centre-sud de la province répond à une volonté d'afficher une appartenance à la foi catholique et à la culture française d'une manière discrète. Cette hiéronymie supplée commodément au recours à l'hagionymie.

\section{Figure 2 Hiéronymie méridionale}

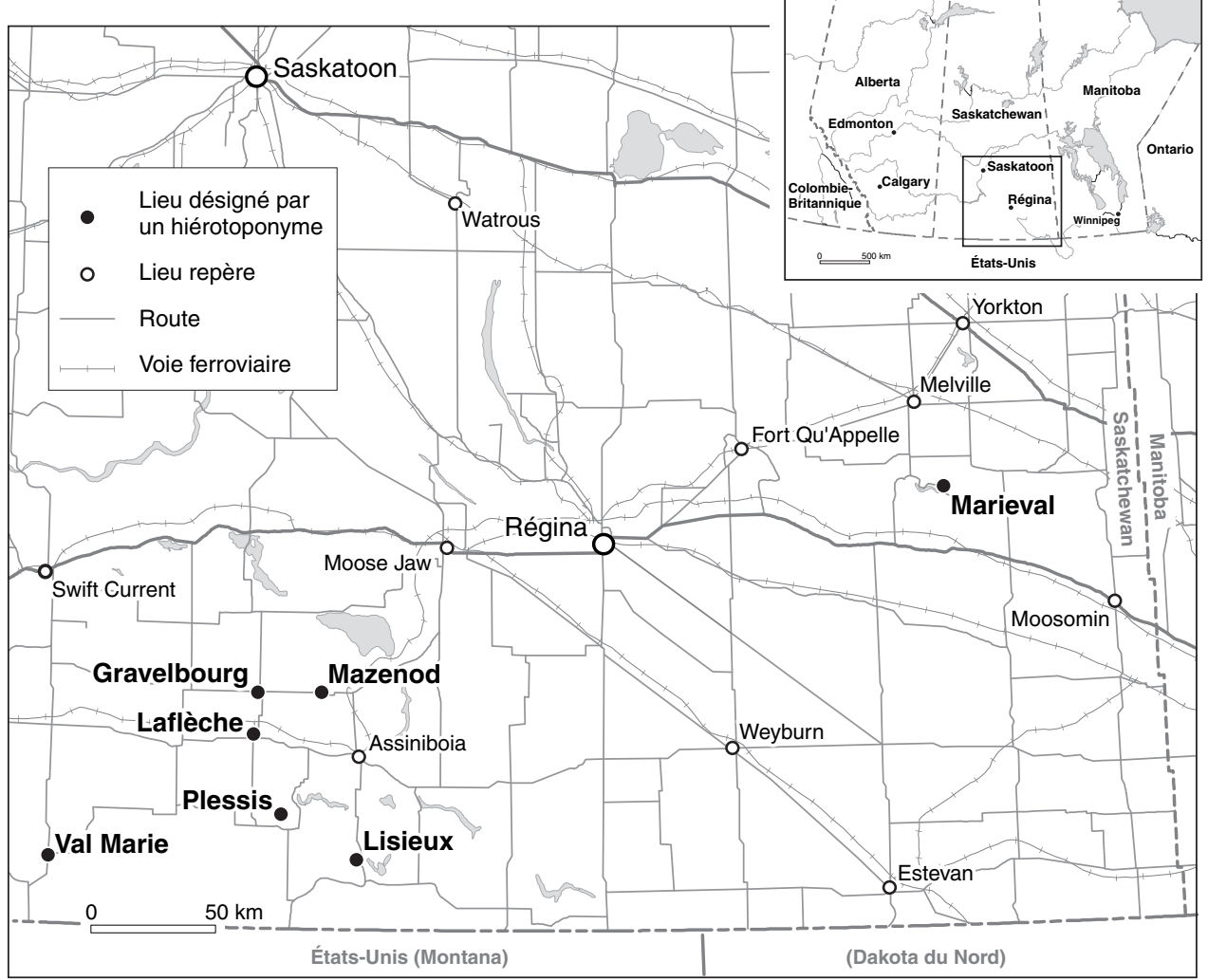

\section{Conclusion}

Produit d'une culture, porté par une langue, élément majeur du paysage linguistique, le nom de lieu est indispensable aux déplacements et à leur évocation. Bien que la fonction locative soit à l'origine même de l'existence du nom de lieu, le toponyme ne répond pas toujours qu'à la seule nécessité de localisation. Il est une composante identitaire parmi les plus intimes d'une collectivité linguistique (Lapierre, 2002). Il peut être symboliquement chargé. L'existence d'une influence symbolique du paysage linguistique sur la vitalité ethnolinguistique des groupes minoritaires a pu être clairement établie (Landry et Bourhis, 1997). Elle agit tel un marqueur de l'état du pouvoir relatif et du statut respectif des groupes linguistiques qui occupent un même territoire. 
Si le toponyme se charge symboliquement, il peut être élevé au rang d'emblème ou devenir référent identitaire. Dans nombre de cas, se superpose, de manière plus ou moins consensuelle à la fonction locative du nom de lieu, une «emblématisation» ou une mythification du toponyme à travers la célébration d'un lieu (Domrémy, Vimy) ou encore d'une personnalité qui fait exemple (Plessis, St-Louis, Lacordaire). La mythification «ajoute à la notoriété et à l'exemplarité la transcendance historique, la sublimation, l'unanimité» (Boyer, 2008: 3). Le toponyme acquiert en pareil cas une fonction de promotion patrimoniale. Autre cas de figure: la fonction identitaire. Le nom de lieu s'affiche alors comme le symbole d'une revendication, d'une affirmation nationale ou d'une lutte contre la confiscation d'usages sociolinguistiques.

Dans une province où l'hagionymie ne figure pas au nombre des modes populaires de désignation des lieux et à une époque où la minorité francophone fortement affaiblie sur le plan démographique se trouvait repoussée jusque dans ses retranchements, les conditions ne se présentaient pas comme étant favorables à la création d'une toponymie d'affirmation identitaire. En revanche, une hiéronymie supplétive se prêtait bel et bien à une promotion patrimoniale. 


\section{Bibliographie}

ADAM, Francine (2008) Des noms et des lieux. La médiation toponymique au Québec et en Acadie du Nouveau-Brunswick. Paris, Université Paris IV, thèse de doctorat non publiée.

BARBER, Marilyn (1972) Introduction. Dans James S. Woodsworth Strangers within our gates; Or coming Canadians. Toronto, University of Toronto Press.

BARRY, Bill (1997) People places: Saskatchewan and its names. Regina, Canadian Plains Research Center.

BERG, Lawrence et KEARNS, Robin A. (1996) Naming as norming: "race", gender, and the identity politics of naming places Aotearoa/New Zealand. Environment and Planning D: Society and Space, vol. 14, $\mathrm{n}^{\circ} 1$, p. 99-122.

BOYER, Henri (2008) Fonctionnements sociolinguistiques de la dénomination toponymique. Mots. Les langages du politique, no86, p. 2-11.

CARRIÈRE, Gaston (1958) Essai de toponymie oblate canadienne. Revue de l'Université d'Ottawa, no28, p. 364-394, p. 522-531.

CHABOT, Adrien (1961) Aperçu historique de Ferland, Sask. Gravelbourg, Saskatchewan

CHOQUETTE, Robert (1995) The Oblate assault on Canada's Northwest. Ottawa, University of Ottawa Press.

DE LA SOUDIÈRE, Martin (2004) Lieux dits: nommer, dé-nommer, re-nommer. Ethnologie française, vol. 34, nº 2, p. 67- 77.

DORION, Henri et BEAUPRÉ, Pierre (1994) Noms et lieux du Québec: dictionnaire illustré. Québec, Commission de toponymie du Québec.

CANADIAN PERMANENT COMMITTEE ON GEOGRAPHICAL NAME/COMITÉ PERMANENT CANADIEN DES NOMS GÉOGRAPHIQUES (1998) Gazetteer of $\mathrm{Ca}$ nada. Répertoire toponymique du Canada. Saskatchewan (4 ${ }^{\text {th }}$ ed.). Ottawa, Centre for Topographic Information/ Centre d'information topographique.
GIRAUT, Frédéric et HOUSSAY-HOLZSCHUCH, Myriam (2008) Néotoponymie: formes et enjeux de la dénomination des territoires émergents. L'Espace Politique, vol. 5, no 2. [En ligne.] http://espacepolitique.revues.org/index161.html

HUEL, Raymond (1978) The Public school as a guardian of Anglo-Saxon traditions: The Saskatchewan experience, 1913-1918. Dans Martin L. Kovacs (dir.) Ethnic Canadians: Culture and Education. Regina, Canadian Plains Studies, no8, p. 295-303.

HUEL, Raymond (1983a) Les Canadiens français et le débat sur la question des langues en 1918. Dans Perspectives sur la Saskatchewan française. Regina, Société historique de la Saskatchewan, p. 313-330.

HUEL, Raymond (1983b) Mgr Olivier-Elzéar Mathieu, protecteur des intérêts francocatholiques en Saskatchewan. Dans Perspectives sur la Saskatchewan française. Regina, Société historique de la Saskatchewan, p. 202-218.

JENSON, Jane (1993) Naming nations: nationalisms in Canadian public discourse, Canadian Review of Sociology and Anthropology, vol.30, n² 2, p. 337-358.

LALONDE, André (1983) Les Canadiens Français de l'Ouest, espoirs, tragédies, incertitudes. Dans Dean Louder et Éric Waddell (dir.) Du continent perdu à l'archipel retrouvé: le Québec et l'Amérique française. Québec, Presses de l'Université Laval, p. 81-95.

LANDRY, Rodrigue et BOURHIS, Richard (1997) Linguistic landscape and ethnolinguistic vitality: an empirical study. Journal of Language and Social Psychology, vol. 16, $\mathrm{n}^{0} 1$, p. 23-49.

LAPIERRE, André (2002) Le toponyme comme marqueur identitaire en Amérique française. Dans Pierre Lanthier et Pierre Savard (dir.) Constructions identitaires et pratiques sociales: actes du colloque en hommage à Pierre Savard tenu à l'Université d'Ottawa les 4, 5, 6 octobre 2000. Ottawa, Presses de l'Université d'Ottawa. 
LAPOINTE, Richard et TESSIER, Lucille (1986) Histoire des Franco-Canadiens de la Saskatchewan. Regina, Société historique de la Saskatchewan.

LÉONARD, Carol (2010) Mémoire des noms de lieux d'origine et d'influence françaises en Saskatchewan. Répertoire toponymique. Québec, Éditions GID.

LIGHT, Duncan, NICOLAE, Ion et SUDITY, Bogdan (2002) Toponymy and the Communist city: Street names in Bucharest, 1948-1965. GeoJournal, vol. 56, nº 2 , p. 135-144.

MAGUIRE, T.M. (1906) North Central Inspectoral Division - T.M. Maguire's Report, Manitoba. Report of the Department of Education.

MORICE, Adrien-Gabriel (1921-1923) Histoire de l'Église catholique dans l'Ouest canadien, du lac Supérieur au Pacifique (1659-1915). Saint-Boniface, Chez l'auteur.

MORICE, Adrien-Gabriel (1928) Histoire de l'Église catholique dans l'Ouest canadien, du Lac Supérieur au Pacifique. Winnipeg, Chez l'auteur (quatrième édition).

NASH, Catherine (1999) Irish placenames: postcolonial locations. Transactions of the Institute of British Geographers, vol. 24, p. 457-480.

PAINCHAUD, Robert (1986) Un rêve français dans le peuplement de la Prairie. Saint-Boniface, Manitoba, Éditions des Plaines.

ROYER, Albert-Marie (1908) Excursion d'un missionnaire en 1907. Clermont-Ferrand, Imprimerie Moderne A. Dumont.

THOMPSON, John Herd (1978) The harvests of war: the Prairie West, 1914-1918. Toronto, McClelland and Stewart.

WOERHLING, Jean-Marie (1998) Conclusions générales. Dans Solange Wydmusch (dir.) La toponymie, un patrimoine à préserver. Paris, L'Harmattan, p. 159-169. 